\title{
Spiral T1 Spin-Echo for Routine Postcontrast Brain MRI Exams: A Multicenter Multireader Clinical Evaluation
}

\author{
(D) M.B. Ooi, (D) Z. Li, (DR.K. Robison, (DD. Wang, (D)A.G. Anderson III, (D)N.R. Zwart, (D)A. Bakhru, (D). Nagaraj, (D). Mathews, \\ (D) S. Hey, (D).J. Koonen, (DI.E. Dimitrov, (D) H.T. Friel, (D) Q. Lu, (D) M. Obara, (DI. Saha, (D) H. Wang, (D). Wang, (D). Zhao, \\ (D) M. Temkit, DH.H. Hu, (D)T.L. Chenevert, (D). Togao, DJ.A. Tkach, DU.D. Nagaraj, DM.C. Pinho, (D)R.K. Gupta, \\ (D).E. Small, (D)M.M. Kunst, (D).P. Karis, DJ.B. Andre, (D).H. Miller, (D) N.K. Pinter, and (DJ.G. Pipe
}

\begin{abstract}
BACKGROUND AND PURPOSE: Spiral MR imaging has several advantages compared with Cartesian MR imaging that can be leveraged for added clinical value. A multicenter multireader study was designed to compare spiral with standard-of-care Cartesian postcontrast structural brain MR imaging on the basis of relative performance in 10 metrics of image quality, artifact prevalence, and diagnostic benefit.
\end{abstract}

\begin{abstract}
MATERIALS AND METHODS: Seven clinical sites acquired 88 total subjects. For each subject, sites acquired 2 postcontrast MR imaging scans: a spiral 2D T1 spin-echo, and 1 of 4 routine Cartesian 2D T1 spin-echo/TSE scans (fully sampled spin-echo at 3T, 1.5T, partial Fourier, TSE). The spiral acquisition matched the Cartesian scan for scan time, geometry, and contrast. Nine neuroradiologists independently reviewed each subject, with the matching pair of spiral and Cartesian scans compared side-by-side, and scored on 10 image-quality metrics (5-point Likert scale) focused on intracranial assessment. The Wilcoxon signed rank test evaluated relative performance of spiral versus Cartesian, while the Kruskal-Wallis test assessed interprotocol differences.
\end{abstract}

RESULTS: Spiral was superior to Cartesian in 7 of 10 metrics (flow artifact mitigation, SNR, GM/WM contrast, image sharpness, lesion conspicuity, preference for diagnosing abnormal enhancement, and overall intracranial image quality), comparable in 1 of 10 metrics (motion artifacts), and inferior in 2 of 10 metrics (susceptibility artifacts, overall extracranial image quality) related to magnetic susceptibility $(P<.05)$. Interprotocol comparison confirmed relatively higher SNR and GM/WM contrast for partial Fourier and TSE protocol groups, respectively $(P<.05)$.

CONCLUSIONS: Spiral 2D T1 spin-echo for routine structural brain MR imaging is feasible in the clinic with conventional scanners and was preferred by neuroradiologists for overall postcontrast intracranial evaluation.

ABBREVIATIONS: Cart = Cartesian; IQ = image quality; NA = not applicable; SE = spin-echo; TSE = turbo spin-echo

$\mathbf{S}$ tructural T1-weighted sequences are a fundamental component of routine postcontrast brain MR imaging examinations. These contrast-enhanced images are used for the diagnostic detection and evaluation of abnormal enhancement, including tumors, infections, and inflammatory diseases. Cartesian 2D T1 spin-echo (SE) is widely used as the standard-of-care, though it is relatively slow due to its single phase-encode per shot $k$-space

Received October 21, 2019; accepted after revision December 10

From Philips Healthcare (M.B.O., I.E.D., H.T.F., Q.L., H.W., Y.W., Y.Z.), Gainesville, Florida; Barrow Neurological Institute (Z.L., A.G.A., N.R.Z., J.P.K.), Phoenix, Arizona; Mayo Clinic (D.W., J.G.P.), Rochester, Minnesota; Phoenix Children's Hospital (R.K.R., M.T., H.H.H., J.H.M.), Phoenix, Arizona; DENT Neurologic Institute (N.K.P.), Buffalo, New York; Philips Healthcare (A.B., S.N., T.M.), Bangalore, India; Philips Healthcare, (S.H., J.J.K.), Best, the Netherlands; Philips Healthcare (M.O.), Tokyo, Japan; Philips Healthcare (I.S.), Gurgaon, India; University of Michigan (T.L.C.), Ann Arbor, Michigan; Kyushu University Hospital (O.T.), Kyushu, Japan; Cincinnati Children's Hospital (J.A.T., U.D.N.), Cincinnati, Ohio; University of Texas Southwestern Medical Center (M.C.P.), Dallas, Texas; Fortis Memorial Research Institute (R.K.G.), Gurgaon, India; Lahey Hospital and Medical Center (J.E.S., M.M.K.), Burlington, Massachusetts; and University of Washington (J.B.A.), Seattle, Washington. coverage, and is not compatible with parallel imaging due to strong free-induction decay artifacts from the refocusing radiofrequency pulse. Two routine speed-up options include Cartesian 2D T1-SE with partial-Fourier $k$-space coverage, but at the cost of reduced SNR; alternatively, Cartesian 2D T1 turbo spin-echo

Paper previously presented, in part, as an oral scientific presentation at: Annual Meeting of the Radiological Society of North America, December 1-6, 2019; Chicago, Illinois; and an oral scientific presentation at: Annual Meeting of the International Society of Magnetic Resonance in Medicine, June 16-21, 2018; Paris, France.

This work is funded, in part, by Philips Healthcare (Z.L., R.K.R., D.W., A.G.A., N.R.Z., J.G.P.).

Please address correspondence to Melvyn B. Ooi, PhD, Barrow Neurological Institute, Keller Center, 350 W Thomas Rd, Phoenix, AZ 85013; e-mail: melvyn.ooi@philips.com

Indicates article with supplemental on-line table.

Indicates article with supplemental on-line photos.

http://dx.doi.org/10.3174/ajnr.A6409 
(TSE) uses an echo-train to cover multiple $k$-space lines per shot, but at the cost of reduced T1 contrast. A challenge common to all Cartesian methods is flow artifacts originating from CSF/blood flow, which manifest in images as a classic coherent ringing in the phase-encoding direction. These artifacts can obscure lesions and lower overall diagnostic confidence. Flow artifacts are further exacerbated in contrast-enhanced scans due to the hyperintense vascular signal.

Spiral MR imaging, a non-Cartesian technique that uses a spiral $k$-space trajectory, ${ }^{1,2}$ provides several advantages over routine Cartesian MR imaging. A primary benefit is scan efficiency due to the longer acquisition duration $(\tau)$ per shot, which enables a concurrent decrease in scan time and/or an increase in SNR. ${ }^{3}$ Spirals are also more robust to artifacts such as flow, foldover aliasing, geometric distortions, and Gibbs ringing; this is due to the spiral trajectory's inherently reduced gradient moments, ${ }^{4}$ center of $k$-space oversampling, nondedicated phase-encoding direction, and incoherent dispersion of unwanted signal changes between spiral arms. ${ }^{5}$ Our spiral implementation is Dixon-based by design; ${ }^{6}$ therefore, the generation of separate water and fat images is another intrinsic benefit. Spiral MR imaging has demonstrated unique advantages across a wide range of applications, including diffusion, ${ }^{7}$ perfusion, ${ }^{8}$ fMRI, ${ }^{9}$ and real-time speech. ${ }^{10}$ These and previous studies were predominantly performed in research settings, on healthy volunteers, required specialized hardware/ reconstruction approaches that are not practical in a highthroughput clinical environment, and/or otherwise described functional (rather than structural) applications with lower imageresolution requirements. Despite its benefits, spiral MR imaging has therefore not gained widespread clinical adoption due to its greater demand on system fidelity (eg, $\mathrm{B}_{0}$ homogeneity, gradient accuracy/precision) and reconstruction complexity.

We present here a multicenter clinical evaluation of spiral MR imaging as an alternative to Cartesian $\mathrm{MR}$ imaging for routine structural brain examinations, based on previous sequence optimization efforts and earlier clinical feasibility results. ${ }^{11-13}$ Spiral 2D T1-SE is compared with Cartesian 2D T1-SE/TSE protocols that are representative of standard-of-care postcontrast brain $\mathrm{MR}$ imaging. A multireader study assessed relative performance based on 10 metrics of image quality, artifact prevalence, and diagnostic benefit.

\section{MATERIALS AND METHODS Industrial Support}

The spiral pulse sequence and reconstruction algorithm is a collaborative "work-in-progress," developed by the Barrow Neurological Institute and Mayo Clinic (Z.L., D.W., N.R.Z., R.K.R., A.G.A., M.B.O., J.G.P.) and funded, in part, by Philips Healthcare.

\section{Patient Population}

This prospective multicenter study was approved by the institutional review board at each site and was Health Insurance Portability and Accountability Act-compliant. Subjects were scanned at 7 clinical sites from April 2018 to November 2018, and informed consent was obtained from each subject (4 sites) or waived (3 sites) as a minimum-risk study to add the proposed sequence at the end of a routine brain MR imaging examination.

Patients undergoing clinically indicated postcontrast routine brain MR imaging examinations were eligible for study inclusion. One hundred subjects were initially imaged. Of these, 12 subjects were excluded due to technical factors, protocol deviations that invalidated scan comparisons, and severe bulk motion. Thus, 88 subjects met the inclusion criteria (54 women, 34 men; mean age, $45 \pm 22$ years) and were subsequently analyzed.

\section{Imaging Methods}

The study was performed on five 3T and three 1.5T MR imaging scanners (Ingenia; Philips Healthcare, Best, the Netherlands) with a standard hardware configuration, using a 15- or 32-channel head coil as per the routine of each site. The spiral 2D T1-SE (spiral-SE) sequence consisted of conventional $90^{\circ}-180^{\circ}$ radiofrequency pulses, followed by a fully sampled spiral-out readout ${ }^{14}$ with acquisition duration $\tau \sim 12 / 22 \mathrm{~ms}$ for 3T/1.5T. Crusher gradients around the $180^{\circ}$ refocusing radiofrequency pulse provided additional flow signal suppression and black-blood contrast. ${ }^{11}$ The spiral implementation is Dixon-based by design, with samples acquired at 2 or 3 unique TEs to encode chemical-shift information, in order to account for the different blurring properties of water/fat. On-line reconstruction ( $\sim 1$ second/slice) was performed using a conjugate gradient algorithm for joint off-resonance deblurring and Dixon-based water/fat separation, ${ }^{6}$ resulting in the intrinsic generation of separate water and fat (along with in-phase, out-of-phase) image series. $\mathrm{A} \mathrm{B}_{0}$ prescan ( $\sim 30$ seconds) was acquired before the spiral scan for use in reconstruction.

Spiral-SE was evaluated against 4 different Cartesian 2D T1SE (Cart-SE) based protocols, which reflected the standard-ofcare of each site. These 4 protocol comparison groups (G1-G4) were G1, fully sampled Cart-SE at 3T, and G2, at 1.5T; alternatively, as speed-up options, sites used G3, Cart-SE with half scan for partial $k$-space coverage, and G4, Cartesian 2D T1-TSE with an echo-train to cover multiple $k$-space lines per shot. Spiral-SE protocols were designed to match each group of G1-G4 for scan time, geometry, and contrast-related parameters as closely as possible, given the constraints of each sequence. Typical parameters for the matching pairs of spiral-versus-Cartesian scans in G1-G4 are shown in the On-line Table.

For each subject, one of the G1-G4 protocols was acquired, resulting in a matching pair of postcontrast spiral-versusCartesian scans for subsequent evaluation. The pair of scans was alternately acquired in forward/reverse order (i.e. spiral followed by Cartesian, or Cartesian followed by spiral) to minimize bias related to delayed enhancement, and added to the end of the routine brain examination to minimize disruption to the clinical routine.

\section{Radiologic Assessment}

Nine neuroradiologists, representing the participating sites, each performed an independent review of all 88 subjects. For each subject, the matching pair of spiral and Cartesian scans was compared side-by-side and scored relative to one another on 10 image quality (IQ) metrics (M1-M10): M1, flow artifact 
mitigation; M2, subjective SNR; M3, GM/WM contrast; M4, image sharpness or delineation of brain structures; M5, individual lesion conspicuity, marked not applicable (NA) if no visible lesions; M6, diagnostic preference for detecting abnormal enhancement, which is the clinical use case of the sequence, in which only diagnostically relevant differences should be considered; M7, overall IQ, intracranial compartment only; M8, motion artifact mitigation; M9, susceptibility artifact mitigation; and M10, overall IQ, extracranial compartment only.

M1-M9 were evaluated in the intracranial compartment only, defined as the brain parenchyma, meninges, and blood vessels. M10 was evaluated in the extracranial compartment only, defined as the skull base and below, as well as the nasal cavity, paranasal sinuses, temporal bone, and orbits. This evaluation was performed to align with the primary clinical use case of the T1-SE/ TSE protocol for intracranial compartment evaluation, while providing a summary metric for extracranial compartment assessment. Each metric was scored on an ordinal 5-point Likert scale: $1=$ Cartesian is much better; $2=$ Cartesian is better; $3=$ Cartesian is comparable with spiral; $4=$ spiral is better; $5=$ spiral is much better. Cartesian and spiral scans were anonymized as "method A" and "method B," though for the spiral scans, both water-only and water + fat Dixon image series were provided for review.

\section{Statistical Analysis}

Statistical analysis was performed in R statistical and computing software (Version 3.5.1; http://www.r-project.org/), and data preparation/plotting, in Python (Version 3.6.7; https://www. python.org/downloads/release/python-367/), with the consultation of a biostatistician (M.T.). Nonparametric statistics were used due to the ordinal scoring data. The one-sample Wilcoxon signed rank test was used to evaluate the relative performance of spiral versus Cartesian. Here, we tested the null hypothesis $\mathrm{H}_{0}$ : $\Delta=3$ versus $\mathrm{H}_{1}: \Delta \neq 3$, where $\Delta$ is the median score over the subject population for a given IQ metric; because a score of 3 = Cartesian is comparable with spiral, null hypothesis rejection $\mathrm{H}_{1}$ suggests that the scoring distribution is not symmetric about 3 but is in favor of either spiral $(\Delta>3)$ or Cartesian $(\Delta<3)$. The Kruskal-Wallis test was used to assess intergroup similarity $\left(\mathrm{H}_{0}\right)$ or difference $\left(\mathrm{H}_{1}\right)$ between the distributions of G1-G4, followed by post hoc analysis with the Dunn test for multiple pair-wise comparisons to determine the relative performances within G1G4. Wilcoxon and Kruskal-Wallis tests were evaluated for each IQ metric M1-M10 using the populations over all subjects ( $n=$ 88 ) and also separated by protocol group (G1-G4, $n=42,20$, 13,13 , respectively). The median reviewer score was used for a given subject. A $P$ value $<.05$ was chosen for statistical significance $\left(\mathrm{H}_{1}\right)$. To control for increased type I error incurred by multiple tests, we adjusted all $P$ values for false discovery rate using the Benjamini-Hochberg procedure. Inter-reviewer agreement was assessed by the $\kappa$ statistic.

\section{RESULTS}

Figures 1-4 show images representative of the multireader scoring results for all 10 metrics. Most images are from G1, which is the most direct comparison of fully-sampled single phaseencoded spiral versus Cartesian and the largest sample size; images from other protocol groups are shown when appropriate. On-line Figures 1-4 contain histograms of all scores for each of M1-M10. Images in Figs 1-4 were selected to reflect the histogram trends in On-line Figs 1-4, respectively, and provide complementary information when viewed together as described below.

Figure 1 and On-line Fig 1 illustrate flow artifact mitigation (M1). The most prominent flow artifacts, either due to CSF or blood flow, are often observed in the posterior cranial fossa (Fig 1). These artifacts severely corrupt Cartesian images (bottom row), manifesting as coherent ringing artifacts in the left-right phase-encoding direction, most commonly around the brain stem, transverse sinuses, sagittal sinuses, carotid arteries, and their branches. Matching spirals (top row) demonstrate significant/consistent flow artifact reduction in these areas. This benefit also extends to the middle cranial fossa (On-line Fig 1A). On-line Fig $1 B$ provides a histogram of all M1 scores and is described in more detail in the legend.

Figure 2 and On-line Fig 2 summarize metrics related to general image attributes: subjective SNR (M2), GM/WM contrast (M3), and image sharpness (M4). Histogram distributions (Online Fig 2) are right-skewed and qualitatively favorable for spiral across all 3 metrics. Subjective SNR (On-line Fig 2A) shows an elevated bar for G3 with a score of 5, corresponding to a higher relative benefit for spiral-versus-Cartesian SE with half scan, compared with protocols G1, G2, G4. Similarly, GM/WM contrast (On-line Fig 2B) shows an elevated bar for G4 with a score of 5 , corresponding to a higher relative benefit for spiral-versusCartesian TSE, compared with protocols G1, G2, G3. Figure 2 highlights these benefits for protocol groups G1 (fully-sampled reference), G3 (elevated SNR), and G4 (elevated contrast).

Figure 3 and On-line Fig 3 demonstrate spiral improvement for clinically related metrics: individual lesion conspicuity (M5) and diagnostic preference for detecting abnormal enhancement (M6), together with a summary score for overall intracranial IQ (M7). Histograms (On-line Fig 3) show right-skewness in favor of spiral across all 3 metrics. Figure 3 highlights the increased diagnostic confidence for spiral in areas with pathologies, including areas with superior suppression of strong flow-ringing artifacts (column 1), removal of more subtle vascular artifacts that may otherwise be confounded with enhancing lesions (columns 2 and 3), and improved lesion visualization due to overall higher SNR (columns 4-6).

Figure 4 and On-line Fig 4 show metrics for motion artifacts (M8), susceptibility artifacts (M9), and overall extracranial IQ (M10). Histograms (On-line Fig 4) show spiral performed comparable with (M8) or poorer than Cartesian (M9, M10). Figure $4 \mathrm{~A}$ shows increased susceptibility artifacts for spiral around areas of artificially/naturally occurring magnetic susceptibility, which can manifest as signal loss and residual blurring. Similar susceptibility artifacts are observed in the extracranial space for spiral, as reflected in the M10 scores in favor of Cartesian (On-line Fig $4 C)$. Further inspection of On-line Fig $4 C$ shows that $\sim 15 \%$ of all cases were given a score of 4 , predominantly by 2 reviewers (orange, green). These 2 reviewers commented that despite the 

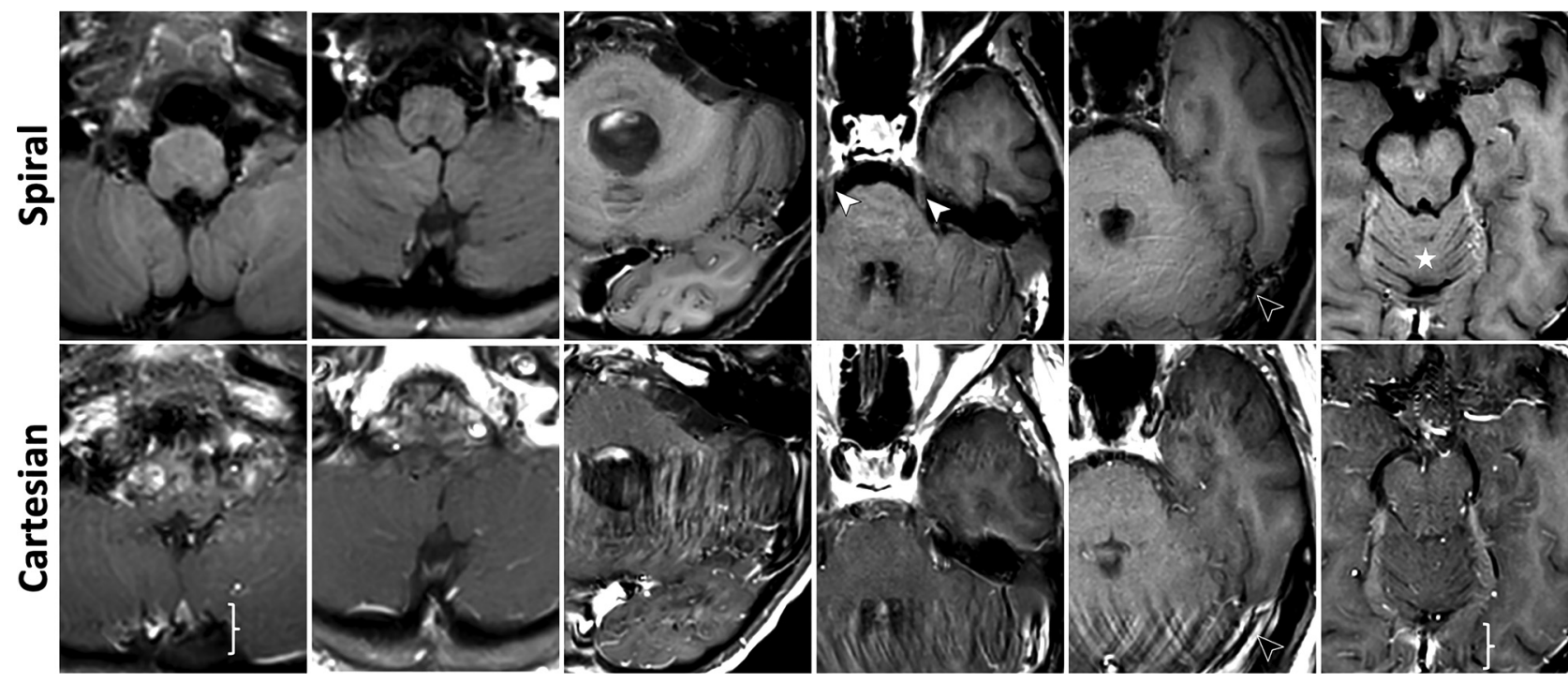

FIG 1. Images illustrating metrics: flow artifact mitigation (MT). See On-line Fig 1 for corresponding histogram of scores. Spirals demonstrate significant and consistent flow artifact mitigation in the inferior slices. All images are taken from Gl. Columns 1-2, Strong flow artifacts around the brain stem and sagittal sinus (brace) in Cartesian are effectively suppressed in spirals, where the previously obscured brain stem and cerebellum are now clearly visualized. Column 3-5, Cartesians exhibit severe flow-ringing artifacts in the phase-encoding direction (left-right) originating from the transverse sinuses and carotid arteries. Spirals produce significantly cleaner images, making visible the cerebellum, anterior temporal lobe, and trigeminal nerves (white arrows); residual flow artifacts in spiral manifest as faint circular ripples emanating from the flow source (black arrows). Column 6, Flow signal in the branches of the carotid, vertebral arteries, and the straight sinus (brace) is well-suppressed in spiral, providing finer structural details of the frontal basal area and cerebellum (star). In Figs 1-4, all spiral images are water-only for brevity.

generally better extracranial IQ of Cartesian, spiral was preferred on a case-by-case basis due to its intrinsic Dixon capability. Figure $4 B$ highlights this advantage, in which spiral water-only images are beneficial for delineating pathologies in/around fat tissue.

Figure 5 summarizes the statistical analysis. The Wilcoxon signed rank test showed that spiral performs better than Cartesian (green) in 7/10 metrics, M1-M7, across all protocol groups; spiral is comparable with Cartesian (blue) in $1 / 10$ metric, M8, across all protocol groups; spiral performs poorer than Cartesian (red) in 2/10 metrics, M9-M10, across all protocol groups, with the exception of M9, G4 (blue). The Kruskal-Wallis with Dunn post hoc analysis provides statistical confirmation of the interprotocol trends observed in the histograms. M2, G3 showed relatively better performance than all other protocols, consistent with the elevated bar in On-line Fig 2A. Similarly, M3, G4 performed better relative to all other protocols, matching the elevated bar in On-line Fig $2 B$. Inter-reviewer agreement scores based on the original 5 -point scale $\left(\kappa_{5 \text { cat }}\right)$ are "slight" to "fair." To further investigate inter-reviewer agreement, all scores were aggregated to a 3-point scale, as either Cartesian is much better or better ( cores $=1,2$, respectively), Cartesian is comparable with spiral (score $=3$, unchanged), or spiral is better or much better (scores $=4,5$, respectively). Inter-reviewer agreement scores based on this 3 -point scale $\left(\kappa_{3 \text { cat }}\right)$ improved to "moderate" or "substantial" for some metrics, suggesting that a source of inter-reviewer difference is due to assessing "better" versus "much better" conditions rather than an entirely different preference between spiral or Cartesian. The Wilcoxon signed rank test was repeated with scores based on the 3-point scale
(On-line Figure 5), which did not change the overall conclusions of Figure 5.

\section{DISCUSSION}

This multicenter clinical evaluation of spiral MR imaging was conducted at sites that represent a variety of MR imaging expert levels, from luminary research institutions to hospitals with limited research support. Furthermore, spiral acquisition and reconstruction were performed on both $3 \mathrm{~T}$ and $1.5 \mathrm{~T}$ scanners with standard hardware configurations. To the best of our knowledge, this multicenter study is the first of its scale and kind to demonstrate the clinical feasibility of spiral MR imaging for routine structural imaging.

Spirals were superior to standard-of-care Cartesian in 7 of 10 assessed metrics. From M1 to M5, the strongest consensus benefit of spiral was flow artifact mitigation, followed by subjective SNR and GM/WM contrast. These cumulative benefits enabled clearer visualization of brain structures and lesions, resulting in better scores in M6-M7 for overall diagnostic preference and intracranial image quality, respectively. The most prominent areas of benefit included the middle cranial fossa, deep gray matter, and posterior cranial fossa. In the middle cranial fossa, reduced flow artifacts around the carotid arteries, in particular, increased diagnostic confidence for temporal lobe pathologies. In deep gray matter, increased SNR, contrast, and lack of flow from the third ventricle and small vessels improved demarcation of the basal ganglia and adjacent white matter tracts. In the posterior cranial fossa, significant flow artifact mitigation from the vertebrobasilar arteries, venous sinuses, fourth ventricle, and foramen magnum dramatically improved image quality of the brain stem, cerebellum, and cranial nerves. Improved anatomic visualization 


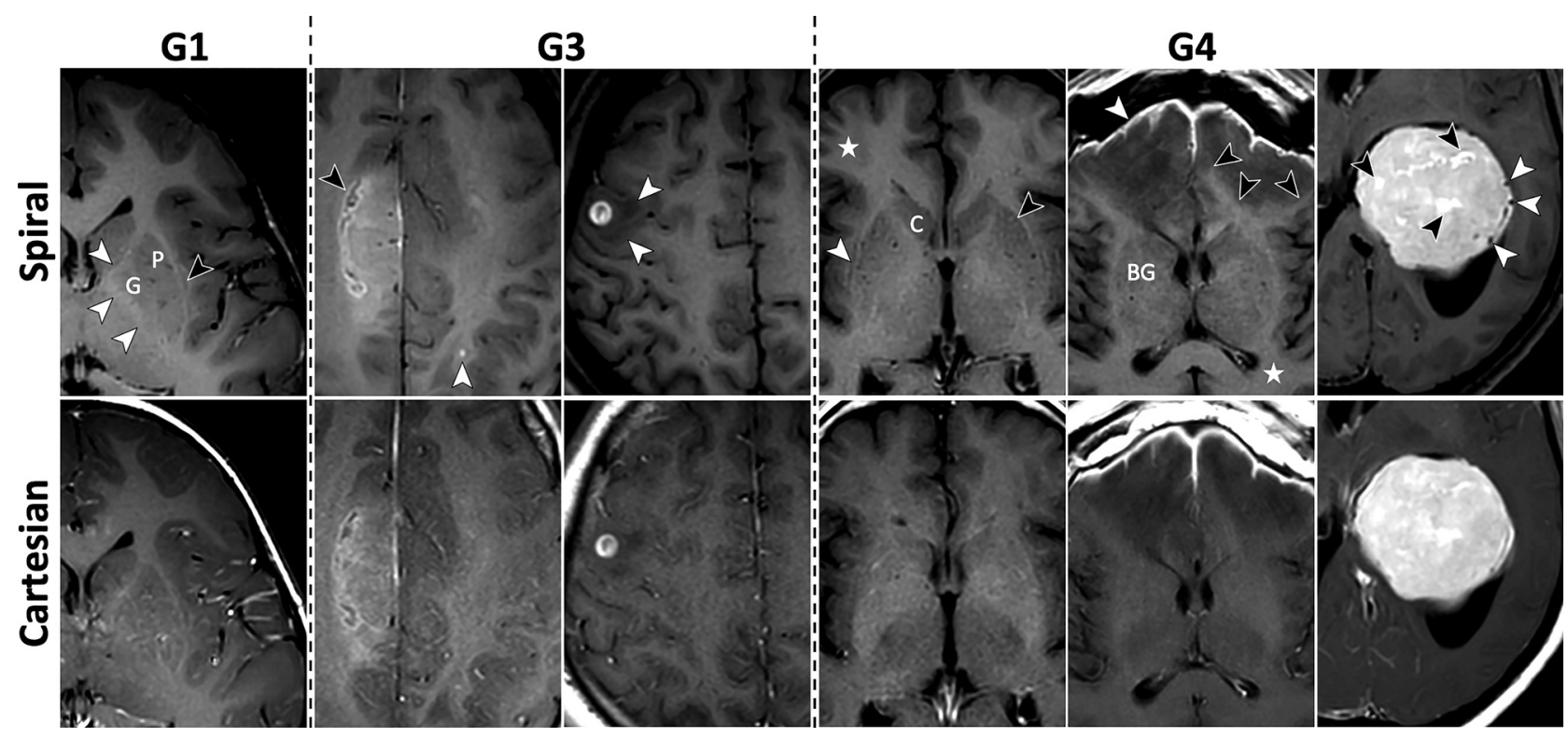

FIG 2. Images for metrics: subjective SNR (M2), GM/WM contrast (M3), and image sharpness (M4). See On-line Fig 2 for corresponding histograms of scores. Column 1, Spiral has higher SNR and GM/WM contrast as demonstrated by the sharper demarcation of the putamen (P) and globus pallidus (G) from surrounding WM tracts. The internal capsule, claustrum, external capsule, and extreme capsule are clearly distinguishable on spiral (white and black arrows), with sharper margins and higher contrast compared with Cartesian. The cortex and subcortical WM show higher contrast as well. Column 2, Grade II isocitrate dehydrogenase (IDH) mutant astrocytoma following radiation therapy is seen in the right hemisphere, showing a more detailed appearance on the spiral (black arrow). The higher spiral SNR also enables confident detection of an enhancing metastasis in the left parietal lobe (white arrow); this lesion is only faintly visible on the Cartesian. Column 3, Spiral shows better defined borders of the vasogenic edema (white arrows) surrounding the contrast-enhancing metastasis. The overall appearance of the lesion is the same on the 2 sequences. Column 4, Spiral demonstrates higher GM/WM contrast, particularly conspicuous by comparing details of the basal ganglia and delineation of the caudate head (C), lateral borders of the anterior putamen (black arrow), and claustrum (white arrow). The cerebral cortex and subcortical WM are better distinguished (star). Column 5, Meningitis (white arrow) and early cerebritis. The extent of the vasogenic edema is easier to assess on spiral due to the higher contrast and sharper boundary with the WM (black arrows). The contrast between WM and deep GM structures (BG) and the cortex (star) is again higher with spiral. Column 6, Spiral GM/WM contrast is generally higher, while Cartesian has less distinguishable GM/WM boundaries. Internal structure of the intraventricular meningioma shows more details, evident by smaller areas of increased enhancement (black arrows) and vascular structures (white arrows) due to spiral flow compensation.

benefits brain imaging in general and, specifically, applications requiring precise anatomic localization such as presurgical planning and image-based morphology. Also, pathologies adjacent to fat tissue were better depicted, exploiting the intrinsic Dixon, suggesting potential utility in head and neck imaging.

The higher scan efficiency of spirals can be leveraged for faster scans and/or increased SNR. In this study, spirals were scan time-matched to Cartesian, resulting in an SNR benefit (M2). With protocol G1 as an example, spirals achieved $~ 150 \%$ higher SNR versus Cartesian. If speed is prioritized, a fullysampled spiral is approximately 4 times faster (scan time $=$ 54 seconds) with $\sim 75 \%$ SNR versus Cartesian. If it is SNRmatched, spiral is approximately 2.3 times faster (scan time $=94$ seconds). Two current speed-up options for routine Cart-SE-based protocols come with trade-offs: reduced SNR due to partial $k$-space coverage (G3) and reduced GM/WM contrast due to the longer echo-train (G4). In both cases, a fully sampled, scan time-matched spiral was acquired without these trade-offs. Interprotocol histogram and KruskalWallis analysis confirm this advantage, with spirals in G3 and G4 demonstrating relatively higher scores for subjective SNR (M2) and GM/WM contrast (M3), respectively, compared with the other protocols. Spirals prioritizing speed may benefit fast screening, and emergency department protocols.
Vascular applications are an area where the scan efficiency, flow suppression, and intrinsic Dixon of the spiral may be leveraged for added clinical value. Vessel wall imaging using current $2 \mathrm{D}$ techniques is slow due to high spatial resolution and requires CSF/blood signal suppression and fat suppression for vessels near the scalp-attributes that have been addressed with the proposed spiral 2D T1-SE. Flow suppression also benefits tumor imaging by separating vascular components in tissue, enabling more precise tumor-size assessment and differentiation of thrombosed/flowing components in vascular tumors. Promising early results have also been demonstrated with spiral TOF-MRA. ${ }^{15}$ For structural T1weighted sequences, many protocols prefer $3 \mathrm{D}$ over $2 \mathrm{D}$ scans; a high-resolution isotropic spiral 3D sequence, with the detailed advantages, will be a competitive alternative to current Cartesian $3 \mathrm{D}$ sequences and could eliminate the need for additional fat-suppressed scans as demonstrated for orbit examinations. ${ }^{16}$

Spirals were comparable with Cartesian in 1 of 10 metrics, motion artifacts. The spiral $k$-space trajectory itself is less sensitive to motion. ${ }^{5,9,10}$ However, a confounding factor is spatial misregistration between the $B_{0}$ prescan and the spiral scan due to bulk head motion, which would result in residual blurring. Further study is required to separate these effects, which may also be mitigated with concurrent motion-tracking $/ \mathrm{B}_{0}$ mapping ${ }^{17}$ or self- $\mathrm{B}_{0}$ techniques. ${ }^{18}$ 


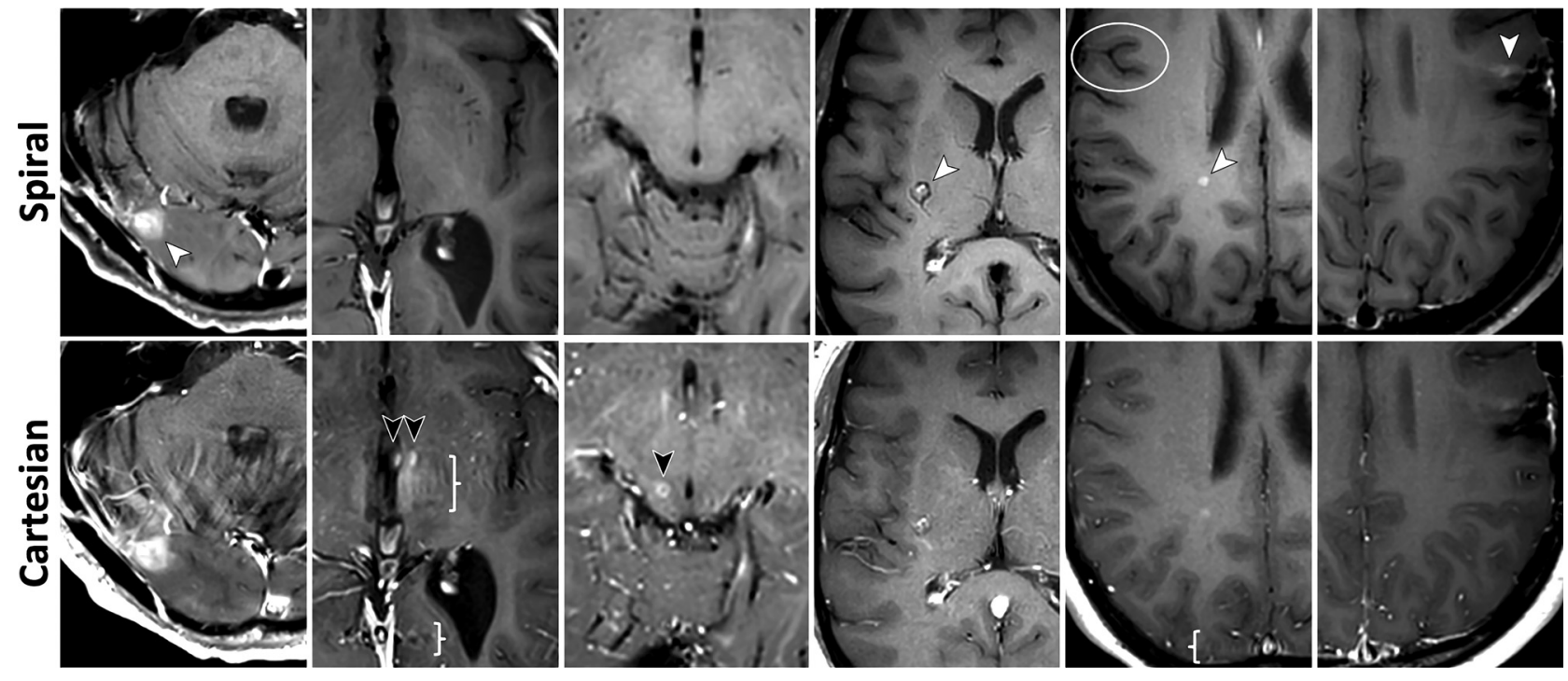

FIG 3. Images for metrics: individual lesion conspicuity (M5), diagnostic preference for detecting abnormal enhancement (M6), and overall intracranial IQ (M7). See On-line Fig 3 for corresponding histograms of scores. All images are taken from Gl. Column 1, Enhancing lesion (white arrow) and surrounding area are better evaluated due to spiral flow artifact reduction. Columns 2 and 3, Misleading hyperintense vascular artifacts (black arrows) are removed in spiral, along with flow-ringing artifacts (braces). Column 4, Cavernous hemangioma in the right basal ganglia (white arrow). The peripheral vascular component is well-separated from the central, contrast-enhancing part by spiral flow suppression. Column 5, An enhancing demyelinating lesion (white arrow) is better depicted on spiral. The spiral was acquired before the Cartesian in this case; thus, the difference is not a result of delayed enhancement. Better delineation of the cortex and higher GM/WM contrast are seen again (circle), along with removal of flow ringing (brace). Column 6, Postsurgical residual enhancement (white arrow) has higher signal and better delineation in spiral, which increases diagnostic confidence.

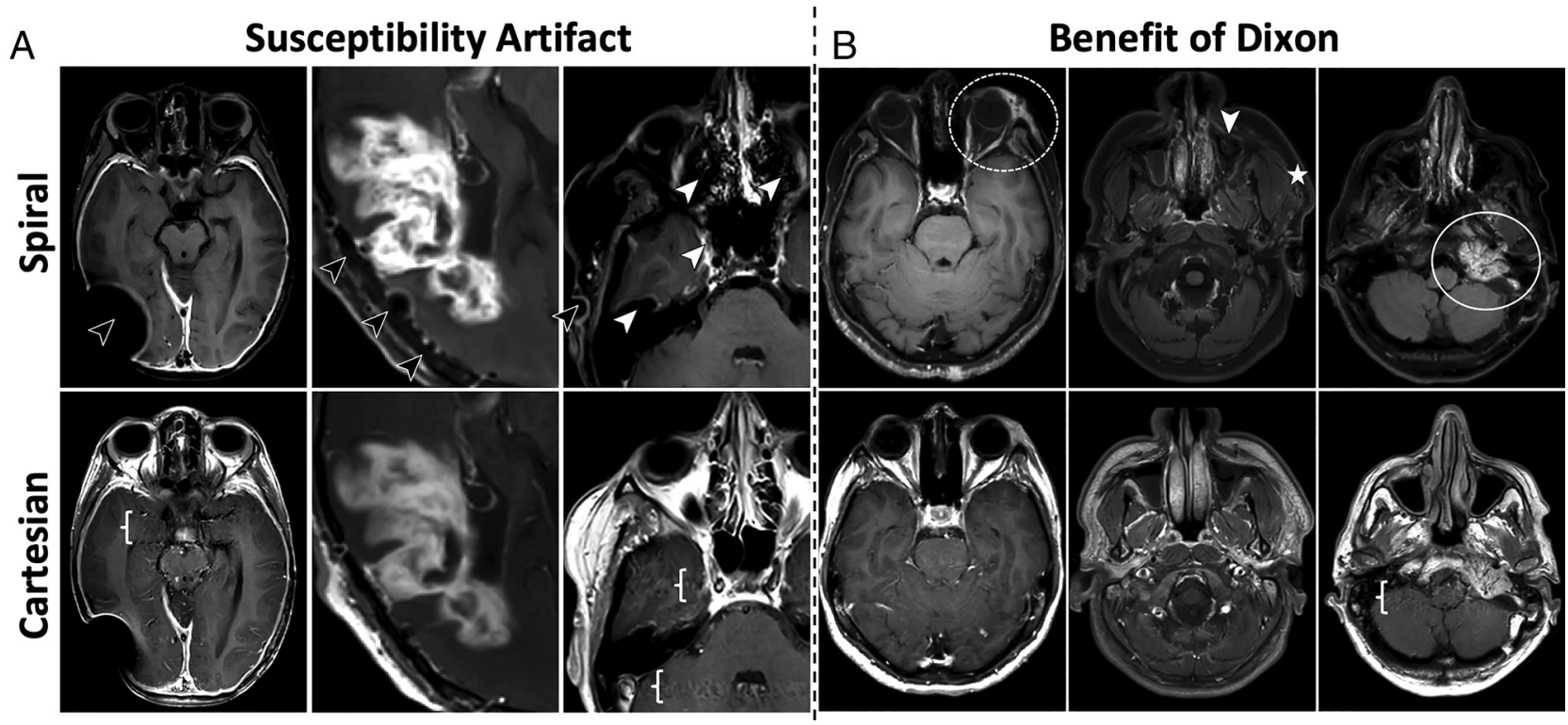

FIG 4. Images for metrics: motion artifacts (M8), susceptibility artifacts (M9), and overall extracranial IQ (M10). See On-line Fig 4 for corresponding histograms of scores. $A$, More pronounced susceptibility artifacts in spirals, appearing as signal voids and residual blurring. These occur in areas where the $B_{0}$ field changes rapidly, such as around metallic implants, surgical clips, and resections (black arrows), as well as air/tissue interfaces in the nasal cavity and sinuses (white arrows). Spiral benefits can be seen in the more detailed internal structure of the enhancing area and flow artifact suppression (braces). B. Extracranial examples that benefit from the intrinsic Dixon water/fat separation of the spiral. Column 4, Spiral shows increased conspicuity of left periorbital inflammatory stranding (dashed circle). Column 5, Sinus and soft-tissue pathology, where spiral increases the visibility of asymmetric inflammatory stranding in the left masticator space (star), though susceptibility artifacts limit visualization of the left maxillary sinus retention cyst (white arrow), while the right maxillary sinus fluid is seen with both techniques. Column 6, Left glomus jugulare tumor (white circle), where spiral highlights the enhancing tumor and separates it from the adjacent fat, while also mitigating posterior fossa flow artifacts (brace). 


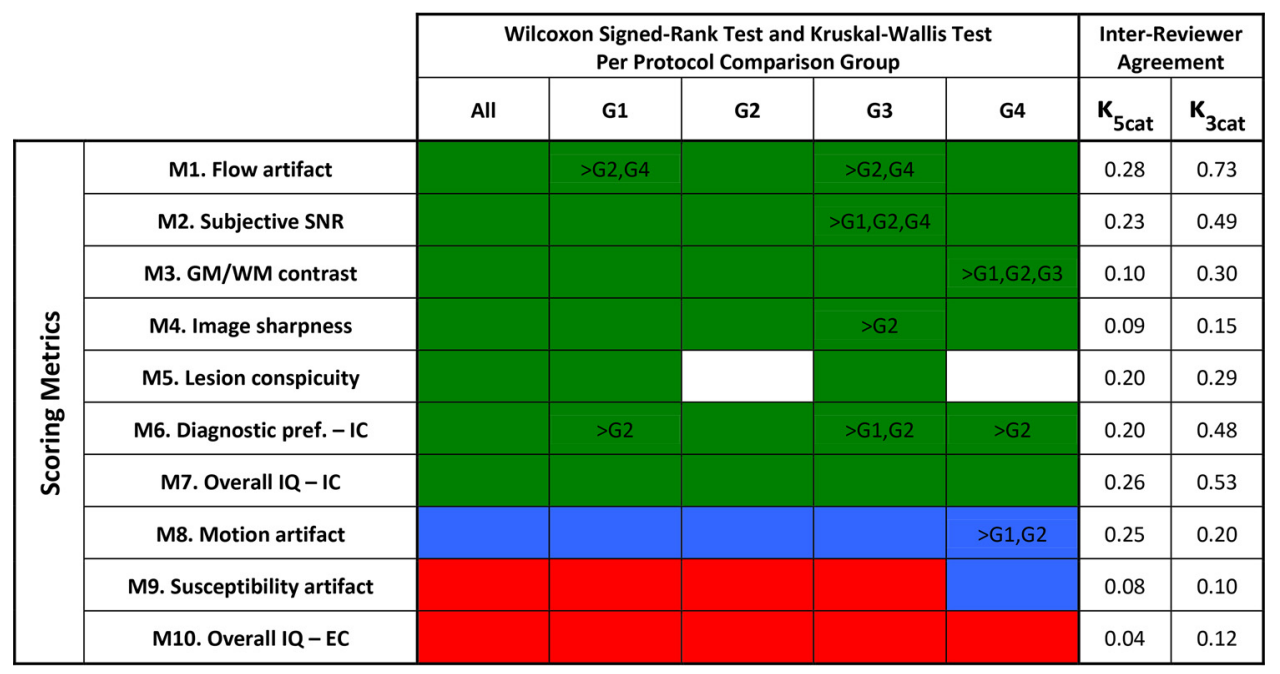

FIG 5. Statistical test results for all assessed metrics (rows: M1-M10), over all protocol groups (columns: All = all cases, G1-G4). Colors denote Wilcoxon signed rank test results: Green indicates that spiral is better than Cartesian ( $P$ value $<.05 ; \Delta>3)$; blue, spiral is comparable with Cartesian ( $P$ value $>$.05); and red, Cartesian is better than spiral $(P$ value $<.05 ; \Delta<3)$. Text within colored cells summarizes Kruskal-Wallis and Dunn test results for interprotocol comparisons. If a row contains text, Kruskal-Wallis resulted in a difference in relative performances between Gl-G4 ( $P$ value $<.05)$; otherwise there was no significant difference between protocols. The text itself summarizes Dunn multiple pair-wise comparison results. For example, for M2, there was a significant difference between G1-G4, where $\mathrm{G} 3$ scores were relatively higher than $\mathrm{G} 1, \mathrm{G} 2$, G4. Similarly for M3, G4 scores were relatively higher than G1, G2, G3. Noncolored cells in M5 are groups with insufficient samples ( $n \leq 5$ ) because subjects without lesions were excluded (marked NA). Inter-reviewer agreement is reported for $\kappa_{5 \text { cat }}$ and $\kappa_{3 \text { cat. }}$ IC indicates intracranial compartment; EC, extracranial compartment; pref, preference; $\kappa_{5 \text { cat }}, \kappa$ statistic for 5 -point scale; $\kappa_{3 \text { cat }}, \kappa$ statistic for 3 -point scale.

Spiral performed poorer than Cartesian in 2 of 10 metrics, both related to magnetic susceptibility in areas where the $B_{0}$ map changes rapidly or is not well-defined. Signal voids and residual blurring can occur in these areas; while these susceptibility artifacts were readily recognizable and did not mimic pathology, diagnostic confidence in artifactual regions may be reduced. Imaging in areas of large magnetic susceptibility is a technical challenge for spiral due to its longer acquisition duration $\tau$, which increases the sensitivity of the technique to offresonances. Shortening $\tau,{ }^{10}$ especially when imaging near metal implants, is one solution, though at the cost of scan efficiency. For maintaining a longer $\tau$ while mitigating susceptibility artifacts, solutions include improved system characterization ${ }^{7,19}$ and alternative deblurring/autofocusing algorithms. ${ }^{20}$

There were several limitations to our study. While the reviewers were blinded to the spiral and Cartesian scan names for scoring, the images themselves contained potentially distinguishable characteristics such as flow artifacts that manifested as swirls in spiral-versus-ringing in Cartesian due to their inherently different $k$-space trajectories. Such features could make each method identifiable to a trained neuroradiologist. Furthermore, spiral images included additional features such as Dixon (water/fat separation) and crusher gradients (flow signal suppression), which could represent a source of sequence identification and/or potential bias.

Regarding Dixon, we elected to distinguish this as an intrinsic benefit of our spiral design and so provided both spiral wateronly and water+fat Dixon image series for review. An alternative approach would be acquisition of Cartesian Dixon with matching Dixon image series. However, this approach would have required changing the standard-of-care for the Cartesian approach, and the added duration would have been impractical for the clinical workflows in this multicenter study. For example, a protocol G1 Cartesian scan time of 3:35 minutes would have doubled to 7 minutes with 2-TE Dixon; paired with a spiral scan time matched to 7 minutes, the result is an additional 14 minutes per subject.

Regarding crusher gradients, this option is not commercially available with the vendor's Cart-SE. The spiral-SE uses a spiralout readout, which enables the insertion of crusher gradients with a minimum increase in TE. On the other hand, insertion of crusher gradients into the Cart-SE would further increase TE (and TR), resulting in reduced SNR and GM/WM contrast. To avoid these trade-offs and to compare with standard-of-care, we therefore did not add crusher gradients to the Cart-SE.

To control for these differences, this study was therefore designed to achieve a fair comparison between spiral and Cartesian protocols by closely matching scan time, geometry (FOV, voxel volume), and contrast-related parameters (TR, TE, flip angle). Standard-of-care Cart-SE/TSE parameters were used as a fixed reference. Spiral SE protocols were then optimized, given these parameter constraints, including the addition of Dixon and crusher gradients within the same scan time, and so forth. Such parameter matching was performed across all protocols and scanners in this multicenter evaluation.

\section{CONCLUSIONS}

Spiral 2D T1-SE for routine structural brain MR imaging is feasible in a clinical population, can be performed at both $3 \mathrm{~T} / 1.5 \mathrm{~T}$ with conventional scanner hardware, and provides several addedvalue benefits compared with standard-of-care Cartesian 2D T1SE/TSE. Spiral was superior to Cartesian in 7 of 10 assessed metrics-with strong consensus benefits to flow artifact mitigation, 
subjective SNR, and GM/WM contrast-and was preferred by neuroradiologists for overall postcontrast intracranial evaluation (M6, M7). Spiral was comparable with Cartesian in 1 of 10 metrics and inferior in 2 of 10 metrics, most notably related to magnetic susceptibility. Ongoing methods development aims to improve the robustness of spiral MR imaging in these areas, while also exploring new clinical applications.

\section{ACKNOWLEDGMENTS}

We thank the following MR imaging technologists for preliminary sequence testing to support protocol optimization: Michael McGranor, Amber Pokorney, Aaron Coover, Efrem Acosta, Soorena Rejaey, Stacey Sullivan Richard, and Noah Briller. We also thank Philips Healthcare for technical support and helpful discussions: Jan Groen, Miha Fuderer, Ad Moerland, Gerald van Ensbergen, Wim Prins, Johan van den Brink, Ashwin Krishnakumar, Kim van de Ven, Liesbeth Geerts-Ossevoort, Patrick Gross, Elizabeth Moore, Jonathan Chia, Marc Van Cauteren, Peter Martin, and Paul Folkers.

Disclosures: Melvyn B. Ooi-RELATED: Grant: Philips Healthcare, Comments: This work was funded, in part, by Philips Healthcare, paid to Barrow Neurological Institute and Mayo Clinic*; UNRELATED: Employment: Philips Healthcare. Zhiqiang Li-RELATED: Grant: Philips Healthcare, Comments: grant and research support.* Ryan K. Robison-RELATED: Grant: Philips Healthcare, Comments: Grant funding from Philips Healthcare related to this work occurred at my previous institution and was prior to the data acquisition, analysis, and manuscript revision specific to this submission.* Dinghui Wang-RELATED: Grant: Philips Healthcare, Comments: Research support from Philips Healthcare.* Ashley G. Anderson-RELATED: Grant: Philips Healthcare, Comments: This work was supported by a grant from Philips Healthcare*; Support for Travel to Meetings for the Study or Other Purposes: Philips Healthcare, Comments: I participated in several conferences, and my travel was funded by grants from Philips Healthcare*; UNRELATED: Employment: Philips Healthcare, Hyperfine Research, Inc. Stock/Stock Options: Philips Healthcare, Hyperfine Research, Inc, Comments: I was employed by Philips Healthcare for some of the time during preparation of this article. At the time of submission, I am an employee of another medical imaging company, Hyperfine Research, Inc. As part of my regular compensation, I have received stock options and participated in employee stock purchase programs. Silke Hey-UNRELATED: Employment: Philips Healthcare Netherlands. Jos Koonen-RELATED: Grant: Eureka, Comments: Eureka Cluster Program ITEA, project STARLIT (16016).* Nandor K. Pinter-UNRELATED: Payment for Lectures Including Service on Speakers Bureaus: Philips Healthcare. James G. PipeRELATED: Grant: Philips Healthcare.* Ivan Dimitrov-UNRELATED: Employment: Philips Healthcare. Quin Lu—UNRELATED: Employment: Philips Healthcare; Stock/ Stock Options: Philips Healthcare. Yansong Zhao-UNRELATED: Employment: Philips Healthcare. Hui Wang-UNRELATED: Employment: Philips Healthcare. Nicholas R. Zwart-RELATED: Grant: Philips Healthcare*; Support for Travel to Meetings for the Study or Other Purposes: Philips Healthcare.* Yi Wang—UNRELATED: Employment: Philips Healthcare. Jalal B. Andre-UNRELATED: Grants/Grants Pending: Philips Healthcare, Comments: research grant.* Thomas L. Chenevert-UNRELATED: Patents (Planned, Pending or Issued): Patent unrelated to this work was licensed to Philips Healthcare, Comments: no money paid. Makoto Obara-OTHER RELATIONSHIPS: I am a Philips Japan employee. *Money paid to institution.

\section{REFERENCES}

1. Ahn CB, Kim JH, Cho ZH. High-speed spiral-scan echo planar NMR imaging-I. IEEE Trans Med Imaging 1986;5:2-7 CrossRef Medline

2. Meyer $\mathrm{CH}, \mathrm{Hu} \mathrm{BS}$, Nishimura DG, et al. Fast spiral coronary artery imaging. Magn Reson Med 1992;28:202-13 CrossRef Medline
3. Pipe JG, Robison RK. Simplified signal equations for spoiled gradient echo MRI. In: Proceedings of the Annual Meeting of the International Society of Magnetic Resonance in Medicine, Stockholm, Sweden. May 1-7, 2010

4. Nishimura DG, Irarrazabal P, Meyer CH. A velocity k-space analysis of flow effects in echo-planar and spiral imaging. Magn Reson Med 1995;33:549-56 CrossRef Medline

5. Pipe JG, Ahunbay E, Menon P. Effects of interleaf order for spiral MRI of dynamic processes. Magn Reson Med 1999;41:417-22 CrossRef Medline

6. Wang D, Zwart NR, Pipe JG. Joint water-fat separation and deblurring for spiral imaging. Magn Reson Med 2018;79:3218-28 CrossRef Medline

7. Wilm BJ, Barmet C, Gross S, et al. Single-shot spiral imaging enabled by an expanded encoding model: demonstration in diffusion MRI. Magn Reson Med 2017;77:83-91 CrossRef Medline

8. Li Z, Schar M, Wang D, et al. Arterial spin labeled perfusion imaging using three-dimensional turbo spin echo with a distributed spiral-in/out trajectory. Magn Reson Med 2016;75:266-73 CrossRef Medline

9. Glover GH. Spiral imaging in fMRI. Neuroimage 2012;62:706-12 CrossRef Medline

10. Lingala SG, Sutton BP, Miquel ME, et al. Recommendations for real-time speech MRI. J Magn Reson Imaging 2016;43:28-44 CrossRef Medline

11. Li Z, Hu HH, Miller JH, et al. A spiral spin-echo MR imaging technique for improved flow artifact suppression in T1-weighted postcontrast brain imaging: a comparison with Cartesian turbo spinecho. AJNR Am J Neuroradiol 2016;37:642-47 CrossRef Medline

12. Ooi MB, Li Z, Wang D, et al. Benefits and challenges of spiral MRI in routine clinical brain imaging: early results. In: Proceedings of the Annual Meeting of the International Society of Magnetic Resonance in Medicine; Paris, France. June 16-21, 2018

13. Ooi MB, Li Z, Robison RK, et al. Spiral T1-SE for routine post-contrast brain MRI: multi-center/reader study results. In: Proceedings of the Annual Meeting of the Radiological Society of North America, Chicago, Illinois. December 1-6, 2019

14. Pipe JG, Zwart NR. Spiral trajectory design: a flexible numerical algorithm and base analytical equations. Magn Reson Med 2014;71: 278-85 CrossRef Medline

15. Wang D, Ooi MB, Zwart NR, et al. Spiral time of flight with sliding slice localized quadratic encoding. In: Proceedings of the Annual Meeting and Exhibition of the International Society of Magnetic Resonance in Medicine, Montreal, Quebec, Canada. May 11-16, 2019

16. Friel $\mathrm{H}, \mathrm{McGranor} \mathrm{M}$, Ooi MB, et al. Clinical application of spiral 3D TFE-IR for postcontrast brain. In: Proceedings of the Annual Meeting of the American Society of Neuroradiology, Vancouver, British Columbia, Canada. June 2-7, 2018

17. Aranovitch A, Vionnet L, Gross S, et al. High-resolution T2*weighted imaging of subcortical brain enhanced by motion and field compensation. In: Proceedings of the Annual Meeting of the International Society of Magnetic Resonance in Medicine. Paris, France. June 16-21, 2018

18. Liu C, Bammer R, Kim DH, et al. Self-navigated interleaved spiral (SNAILS): application to high-resolution diffusion tensor imaging. Magn Reson Med 2004;52:1388-96 CrossRef Medline

19. Robison RK, Li Z, Wang D, et al. Correction of BO eddy current effects in spiral MRI. Magn Reson Med 2019;81:2501-13 CrossRef Medline

20. Man LC, Pauly JM, Macovski A. Improved automatic off-resonance correction without a field map in spiral imaging. Magn Reson Med 1997;37:906-13 CrossRef Medline 